\title{
PERIODIC ORBITS AND NON-INTEGRABILITY IN A COSMOLOGICAL SCALAR FIELD
}

\author{
JAUME LLIBRE ${ }^{1}$ AND CLAUDIO VIDAL ${ }^{2}$
}

\begin{abstract}
We apply the averaging theory of first order to study the periodic orbits of Hamiltonian systems describing an universe filled with a scalar field which possesses three parameters. The main results are the following.

First, we provide sufficient conditions on the parameters of these cosmological model, which guarantee that at any positive or negative Hamiltonian level, the Hamiltonian system has periodic orbits, the number of such periodic orbits and their stability change with the values of the parameters. These periodic orbits live in the whole phase space in a continuous family of periodic orbits parameterized by the Hamiltonian level.

Second, under convenient assumptions we show the non-integrability of these cosmological systems in the sense of Liouville-Arnol'd, proving that cannot exist any second first integral of class $\mathcal{C}^{1}$.

It is important to mention that the tools (i.e the averaging theory for studying the existence of periodic orbits and their kind of stability, and the multipliers of these periodic orbits for studying the integrability of the Hamiltonian system) used here for proving our results on the cosmological scalar field, can be applied to Hamiltonian systems with an arbitrary number of degrees of freedom.
\end{abstract}

\section{IntroduCtion AND STATEMENTS OF MAIN RESUlts}

For a good introduction to the cosmological model here studied we suggest to the reader to look at the paper of Maciejewski et al. [14] and references therein for a detailed deduction and implications about the importance of this model.

The foundation of homogeneous and isotopic cosmological models is the FriedmannRobertson-Walker (FRW) universe, described by the metric

$$
d s^{2}=a(\eta)^{2}\left[-d \eta^{2}+\frac{d r^{2}}{1-K r^{2}}+r^{2} d^{2} \Omega_{2}\right],
$$

where $a$ is the scalar factor, $d^{2} \Omega_{2}$ is the line element on a two-sphere, and we chose to use the conformal time $\eta$. As it is known from the previous metric, the scalar factor represents the relative change in the distance of two points whose spatial coordinates are fixed. It depends only on the time, so that the whole universe is deformed in a homogeneous fashion. Depending on the matter components one obtains various evolution of the scalar factor $a$, as given by the general action

$$
\mathcal{I}=\frac{c^{4}}{16 \pi G} \int\left[\mathcal{R}-2 \Lambda-\frac{1}{2}\left(\nabla_{\alpha} \bar{\psi} \nabla^{\alpha} \psi+V(\psi)+\xi \mathcal{R}|\psi|^{2}\right)-\rho\right] \sqrt{-g} d^{4} x,
$$

where $\mathcal{R}$ is the Ricci scalar, $\Lambda$ is the cosmological constant, $V$ the field's potential, $\xi$ the coupling constant and $\rho$ is the density of the perfect fluid. The potential usually

2000 Mathematics Subject Classification. Primary 34C10, 34C25.

Key words and phrases. periodic orbits, integrability, cosmological scalar fields, averaging theory. 
includes at least a quadratic term $m^{2}|\psi|^{2}$, where $m$ is the so called mass of the field. When $\xi=0$ we say that the field is minimally coupled-it does not uncouple since the determinant of the metric $g$ multiplies the whole Lagrangian density. The case when $\xi=\frac{1}{6}$ is the so called conformal coupling. Under geometric assumptions, the above action can be simplified so that it allows the Hamiltonian approach with the phase variables depending only on conformal time $\eta$. After including an additional matter component $\rho$ is equivalent to considering different energy levels. Namely for $\rho \propto a^{-4}$ (which is the case of radiation) a constant is added to the Hamiltonian, thus imitating its nonzero value. This is the justification for studying the integrability of these systems on a generic energy hypersurface.

As was saw in [14] the general action (2) for conformally coupled scalar fields must includes the following part

(3) $\mathcal{I}=\frac{c^{4}}{16 \pi G} \int\left[\mathcal{R}-2 \Lambda-\frac{1}{2}\left(\nabla_{\alpha} \bar{\psi} \nabla^{\alpha} \psi+\frac{m^{2}}{\hbar}|\psi|^{2}+\frac{1}{6} \mathcal{R}|\psi|^{2}\right)-\frac{\lambda}{4 !}|\psi|^{4}\right] \sqrt{-g} d^{4} x$,

where the additional coupling to gravity through the Ricci scalar $\mathcal{R}$, and a quartic potential term with constant $\lambda$ are considered. After some algebraic manipulations, assuming that the constant angular momentum is null, and under the use of convenient variables the Hamiltonian associated to the action (3) assumes the form

(4) $H=H\left(q_{1}, q_{2}, p_{1}, p_{2}\right)=\frac{1}{2}\left(-p_{1}^{2}+p_{2}^{2}\right)+\frac{1}{2}\left[k\left(-q_{1}^{2}+q_{2}^{2}\right)+m^{2} q_{1}^{2} q_{2}^{2}\right]+\frac{1}{4}\left(\Lambda q_{1}^{4}+\lambda q_{2}^{4}\right)$, with $k \in\{-1,0,1\} K=k|K|$ is associated to the index of curvature of the space), $\lambda, \Lambda, m^{2} \in \mathbb{R}$. Notice that the kinetic part is of natural form, albeit indefinite, and the potential associated is a polynomial of degree four.

In this paper we study the case $k=1$, so the Hamiltonian system is given by

$$
\begin{aligned}
& \dot{q}_{1}=-p_{1}, \\
& \dot{q}_{2}=p_{2}, \\
& \dot{p}_{1}=q_{1}-m^{2} q_{1} q_{2}^{2}-\Lambda q_{1}^{3}, \\
& \dot{p}_{2}=-q_{2}-m^{2} q_{1}^{2} q_{2}-\lambda q_{2}^{3} .
\end{aligned}
$$

As usual the dot denotes derivative with respect to the independent variable $t \in \mathbb{R}$, the time. According to [14] we name (5) the conformal coupled scalar field Hamiltonian systems with three parameters.

The periodic orbits are the most simple non-trivial solutions of a differential system. Their study is of particular interest because the motion in their neighborhood can be determined by their kind of stability. Furthermore, if the system is non-integrable in the sense of Liouville -Arnol'd, the existence of isolated periodic orbits in the energy levels of a Hamiltonian system with multipliers different from 1 is related with the nonexistence of any second first integral of class $\mathcal{C}^{1}$, so the study of these periodic orbits for a differential system becomes relevant.

In general is very difficult to study analytically the existence of periodic orbits and the kind of their stability for a given Hamiltonian system. In this work we use the averaging method of first order to compute periodic orbits and their kind of stability as it was established in [7], see Appendix for a summary of this method. This method allows to find periodic orbits of our cosmological model (5), up to first order in $\varepsilon$, at any non-zero Hamiltonian level as a function of the parameters $\lambda, \Lambda$ and $m$. Roughly speaking, this method reduces the problem of finding periodic solutions of some differential system to the one of finding zeros of some convenient finite dimensional function. In [10] and 
[11] some of us applied these techniques to the Henon-Heiles and to the Yang-Mills Hamiltonians. As we have mention in the abstract the averaging theory for studying the existence of periodic orbits and their kind of stability, and the multipliers of these periodic orbits for studying the integrability of the Hamiltonian system are the tools used here for proving our results on the cosmological scalar field. We remark that these tools can be applied to Hamiltonian systems with an arbitrary number of degrees of freedom.

As we shall see one of the main problems for applying the averaging theory for studying the periodic orbits of a given differential system is to find the changes of variables which allow to write the original differential systems in the normal form for applying the averaging theory. For more details in this direction see the book [22].

Next we will define some subsets of the $(\lambda, \Lambda)$-plane for each nonzero fixed value of $m$ (see Figure 1-2). The straight lines:

$$
\begin{aligned}
& l_{1}: \quad \lambda=-\frac{m^{2}}{3} \\
& l_{2}: \quad \lambda=-m^{2} \\
& l_{3}: \quad \Lambda=-\frac{m^{2}}{3} . \\
& l_{4}: \quad \Lambda=-m^{2} .
\end{aligned}
$$

The regions:

$$
\begin{aligned}
& R_{1}: \quad \lambda<-m^{2}, \Lambda>-m^{2}, \lambda+\Lambda>-2 m^{2} ; \\
& R_{2}: \quad \lambda>-m^{2}, \Lambda<-m^{2}, \lambda+\Lambda<-2 m^{2} ; \\
& R_{3}: \quad \lambda<-\frac{m^{2}}{3}, \Lambda>-\frac{m^{2}}{3}, \lambda+\Lambda>-\frac{2 m^{2}}{3} ; \\
& R_{4}: \quad \lambda>-\frac{m^{2}}{3}, \Lambda<-\frac{m^{2}}{3}, \lambda+\Lambda<-\frac{2 m^{2}}{3} ; \\
& \Omega_{1}: \lambda<-m^{2}, \Lambda>-m^{2}, \lambda+\Lambda<-2 m^{2} ; \\
& \Omega_{2}: \lambda>-m^{2}, \Lambda<-m^{2}, \lambda+\Lambda>-2 m^{2} ; \\
& \Omega_{3}: \quad \lambda<-\frac{m^{2}}{3}, \Lambda>-\frac{m^{2}}{3}, \lambda+\Lambda<-\frac{2 m^{2}}{3} ; \\
& \Omega_{4}: \quad \lambda>-\frac{m^{2}}{3}, \Lambda<-\frac{m^{2}}{3}, \lambda+\Lambda>-\frac{2 m^{2}}{3} .
\end{aligned}
$$

Also we define the half-lines:

$$
\begin{aligned}
& s_{13}=\partial\left(R_{1} \cap R_{3}\right) \cap l_{3} ; \\
& s_{31}=\partial\left(R_{1} \cap R_{3}\right) \cap\left\{\lambda+\Lambda=-\frac{2 m^{2}}{3}\right\} ; \\
& s_{24}=\partial\left(R_{2} \cap R_{4}\right) \cap\left\{\lambda+\Lambda=-2 m^{2}\right\} ; \\
& s_{42}=\partial\left(R_{2} \cap R_{4}\right) \cap l_{1} ; \\
& \tilde{s}_{13}=\partial\left(\Omega_{1} \cap \Omega_{3}\right) \cap\left\{\lambda+\Lambda=-2 m^{2}\right\} ; \\
& \tilde{s}_{31}=\partial\left(\Omega_{1} \cap \Omega_{3}\right) \cap l_{3} ; \\
& \tilde{s}_{24}=\partial\left(\Omega_{2} \cap \Omega_{4}\right) \cap l_{4} ; \\
& \tilde{s}_{42}=\partial\left(\Omega_{2} \cap \Omega_{4}\right) \cap\left\{\lambda+\Lambda=-\frac{2 m^{2}}{3}\right\},
\end{aligned}
$$

where $\partial$ means boundary.

Our main result about the periodic orbits of the Hamiltonian system (5) is summarized as follows.

Theorem 1. For every $m \neq 0$ at every positive Hamiltonian level the conformal coupled scalar field Hamiltonian system (5) has at least

(a1) one periodic orbit if $(\lambda, \Lambda) \in s_{31} \cup s_{42}$;

(a2) two periodic orbits if $(\lambda, \Lambda) \in s_{13} \cup s_{24}$;

(a3) three periodic orbits if $(\lambda, \Lambda) \in\left[R_{1} \backslash\left(R_{1} \cap R_{3}\right)\right] \cup\left[R_{2} \backslash\left(R_{2} \cap R_{4}\right)\right]$;

(a4) four periodic orbits if $(\lambda, \Lambda) \in\left[R_{3} \backslash\left(R_{1} \cap R_{3}\right)\right] \cup\left[R_{4} \backslash\left(R_{2} \cap R_{4}\right)\right]$; 


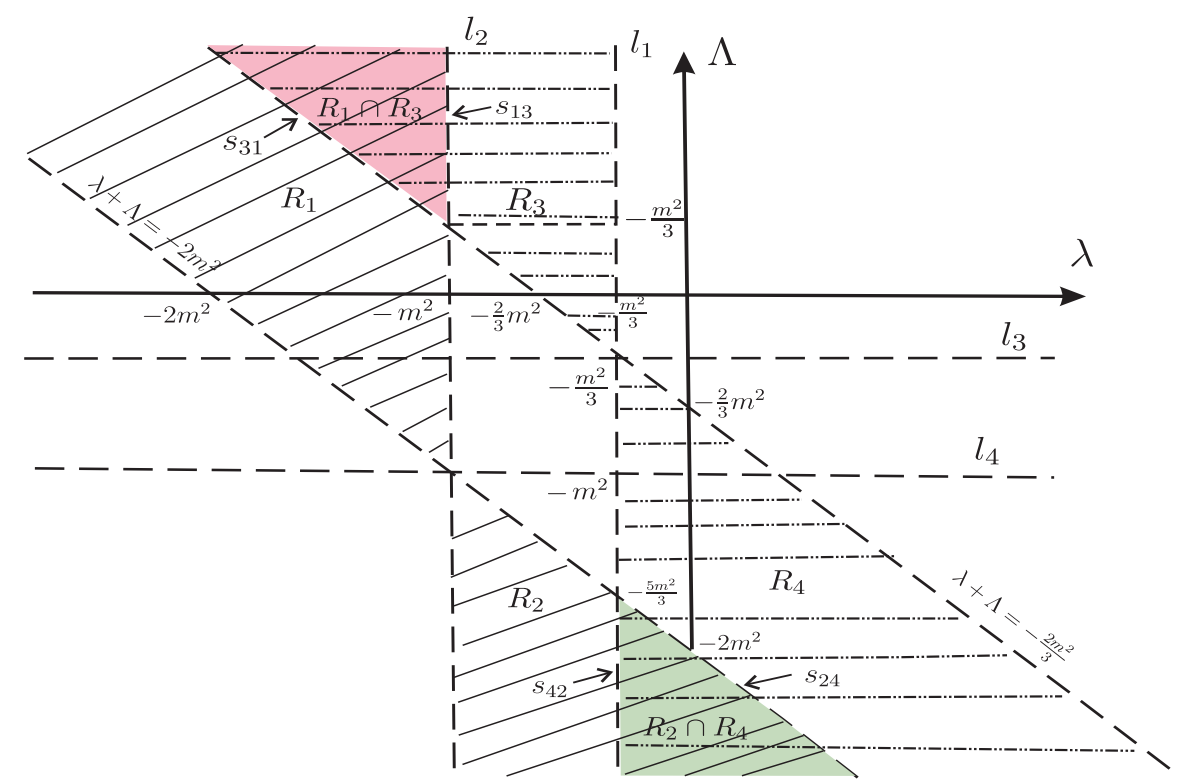

FiguRE 1. Regions $R_{1}, R_{2}, R_{3}, R_{4}$ for $h>0$ and $m \neq 0$.

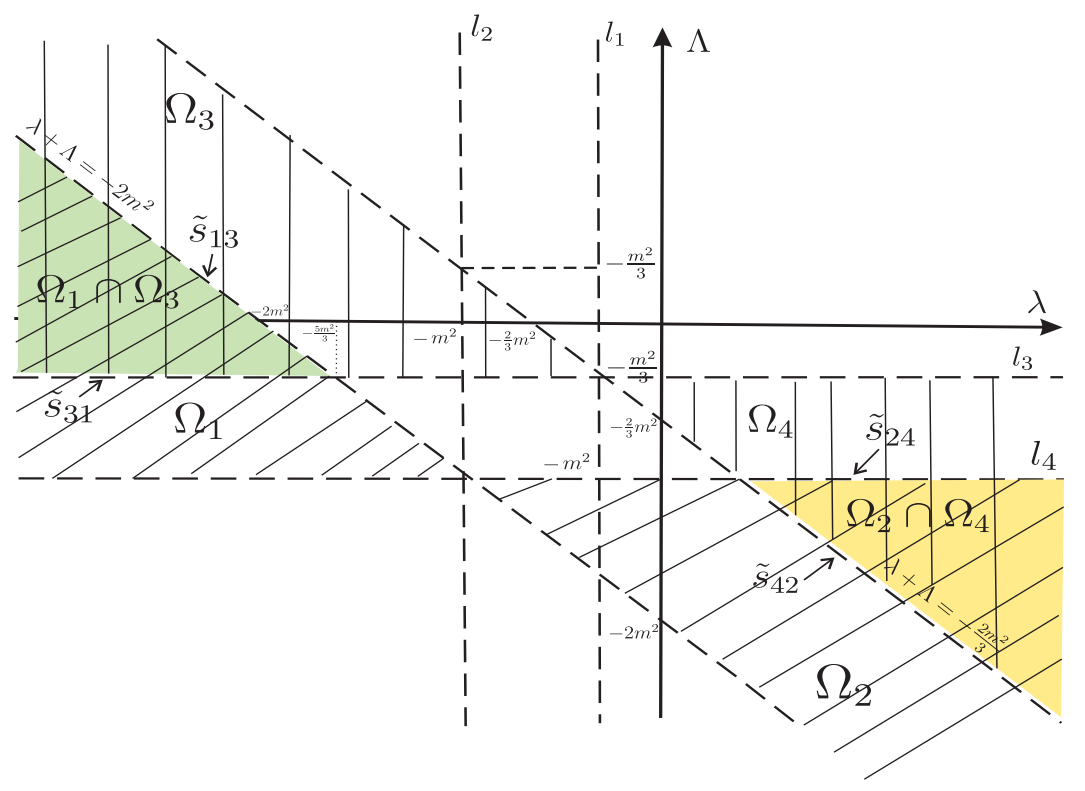

FiguRE 2. Regions $\Omega_{1}, \Omega_{2}, \Omega_{3}, \Omega_{4}$ for $h<0$ and $m \neq 0$.

(a5) five periodic orbits if $(\lambda, \Lambda) \in\left[R_{1} \cap R_{3}\right] \cup\left[R_{2} \cap R_{4}\right]$.

For $m \neq 0$ and for every negative Hamiltonian level the conformal coupled scalar field Hamiltonian system (5) has at least

(b1) one periodic orbit if $(\lambda, \Lambda) \in \tilde{s}_{31} \cup \tilde{s}_{42}$;

(b2) two periodic orbits if $(\lambda, \Lambda) \in \tilde{s}_{13} \cup \tilde{s}_{24}$;

(b3) three periodic orbits if $(\lambda, \Lambda) \in\left[\Omega_{1} \backslash\left(\Omega_{1} \cap \Omega_{3}\right)\right] \cup\left[\Omega_{2} \backslash\left(\Omega_{2} \cap \Omega_{4}\right)\right]$; 
(b4) four periodic orbits if $(\lambda, \Lambda) \in\left[\Omega_{3} \backslash\left(\Omega_{1} \cap \Omega_{3}\right)\right] \cup\left[\Omega_{4} \backslash\left(\Omega_{2} \cap \Omega_{4}\right)\right]$;

(b5) five periodic orbits if $(\lambda, \Lambda) \in\left[\Omega_{1} \cap \Omega_{3}\right] \cup\left[\Omega_{2} \cap \Omega_{4}\right]$.

Theorem 1 is proved in section 2 using the averaging theory.

From Theorem 1 it follows that when $m \neq 0$ (fixed) and $h>0$, we have periodic solutions in our cosmological model for any choice of the parameters $(\lambda, \Lambda) \notin l_{1} \cup l_{2}$. The same conclusion holds for the case $h<0$ if $(\lambda, \Lambda) \notin l_{3} \cup l_{4}$. Thus combining these results we obtain that there are four values of the parameters $(\lambda, \Lambda)$, namely, $P_{1}=\left(-\frac{m^{2}}{3},-\frac{m^{2}}{3}\right) \in l_{1} \cap l_{3}, P_{2}=\left(-m^{2},-\frac{m^{2}}{3}\right) \in l_{2} \cap l_{3}, P_{3}=\left(-m^{2},-m^{2}\right) \in l_{1} \cap l_{4}$ and $P_{4}=\left(-\frac{m^{2}}{3},-m^{2}\right) \in l_{2} \cap l_{4}$, where our arguments do not guarantee the existence of periodic solutions for the Hamiltonian system (5).

The periodic solutions of Theorem 1 are of the form

$$
\begin{array}{ll}
q_{1}(t)=r^{*} \cos (t)+O(\varepsilon), & p_{1}(t)=r^{*} \sin (t)+O(\varepsilon) \\
q_{2}(t)=\rho^{*} \cos \left(-t+\alpha^{*}\right)+O(\varepsilon), & p_{2}(t)=\rho^{*} \sin \left(-t+\alpha^{*}\right)+O(\varepsilon),
\end{array}
$$

where $r^{*}, \rho^{*}, \alpha^{*}$ are functions of the parameters $h, m, \lambda, \Lambda$, and $\varepsilon$ is a small parameter which will be defined later on. In the definition of the following four cases we assume that $m$ and $h$ are fixed with $m h \neq 0$.

Families (I): $h>0$ and $(\lambda, \Lambda) \notin l_{1} \cup l_{2}$. We have two families of periodic solutions (6) generated by a circle on the plane $\left(q_{2}, p_{2}\right)$ with $r^{*}=0, \rho^{*}=\sqrt{h}, \alpha^{*}=$ $\pm \frac{1}{2} \arccos \left(-\frac{2 m^{2}+3 \lambda}{m^{2}}\right)$.

Families (II): $h<0$ and $(\lambda, \Lambda) \notin l_{3} \cup l_{4}$. We have two families of periodic solutions (6) but they are generated by a circle on the $\left(q_{1}, p_{1}\right)$-plane with $r^{*}=\sqrt{-2 h}, \rho^{*}=0, \alpha^{*}=$ $\pm \frac{1}{2} \arccos \left(-\frac{2 m^{2}+3 \Lambda}{m^{2}}\right)$.

Family (III): Either $h>0$ and $(\lambda, \Lambda) \in R_{1} \cup R_{3}$, or $h<0$ and $(\lambda, \Lambda) \in \Omega_{1} \cup \Omega_{2}$. We have one parametric family of periodic solutions generated by the periodic solution (6) with $r^{*}=\sqrt{-\frac{2 h\left(m^{2}+\lambda\right)}{2 m^{2}+\lambda+\Lambda}}, \rho^{*}=\sqrt{\frac{2 h\left(m^{2}+\Lambda\right)}{2 m^{2}+\lambda+\Lambda}}, \alpha^{*}=0$.

Families (IV): Either $h>0$ and $(\lambda, \Lambda) \in R_{2} \cup R_{4}$, or $h<0$ and $(\lambda, \Lambda) \in \Omega_{2} \cup \Omega_{4}$. Here we have two families of periodic solutions generated by the periodic solutions (6) with $r^{*}=\sqrt{-\frac{2 h\left(m^{2}+3 \lambda\right)}{2 m^{2}+3(\lambda+\Lambda)}}, \rho^{*}=\sqrt{\frac{2 h\left(m^{2}+3 \Lambda\right)}{2 m^{2}+3(\lambda+\Lambda)}}, \alpha^{*}= \pm \frac{\pi}{2}$.

It is well known that integrable and non-integrable Hamiltonian systems can have infinitely many periodic orbits. However it is difficult to find a whole family of periodic orbits in an analytical way, specially if the Hamiltonian system is non-integrable. Here we find them up to first order in $\varepsilon$. Once we have shown that at any non-zero Hamiltonian level there exist periodic orbits, we can use these particular periodic orbits to prove our second main result about the non-integrability in the sense of Liouville-Arnol'd of our conformal coupled scalar field Hamiltonian system (5).

Theorem 2. Assume that the conformal coupled scalar field Hamiltonian system (5) satisfies the assumptions of one of the statements of Theorem 1, and denote by $(\sharp)$ this statement. Then, under the assumption of statement ( $\sharp)$,

(a) either the conformal coupled scalar fields Hamiltonian system (5) is LiouvilleArnol'd integrable and the gradients of the two constants of motion are linearly dependent on some points of the periodic orbits found in statement ( $\sharp$ ) of Theorem 1, 
(b) or the conformal coupled scalar fields Hamiltonian system (5) is not LiouvilleArnol'd integrable with any second first integral of class $\mathcal{C}^{1}$.

Theorem 2 is proved in section 3. For a precise definition of the notion of integrability in the sense of Liouville-Arnol'd, see the Appendix. From now on we shall use the definition of integrability in the sense of Liouville-Arnol'd.

We must mention that in general to study the non-integrability of a Hamiltonian system, in our case the existence of an additional second first integral independent of the Hamiltonian, it is very hard, and of course numerically this is not possible to detect, see for instance [2].

In section 4 we characterize the stability or instability of each family of periodic orbits on each Hamiltonian level $h \neq 0$, again using the averaging method. The main result on the stability or instability of the periodic orbits is the following.

Theorem 3. The stability or instability of the families of periodic orbits (6) on each level $h$ of the Hamiltonian system (5) is as follows.

(a) The two families (I) are linearly stable in the regions $I_{1}: \lambda>-m^{2}$ and $I_{3}: \lambda<-3 m^{2}$, and are unstable in the region $I_{2}:-3 m^{2}<\lambda<-m^{2}$.

(b) The two families (II) are linearly stable in the regions $I I_{1}: \Lambda>-\frac{m^{2}}{3}$ and $I I_{3}: \Lambda>-m^{2}$, and are unstable in the region $I I_{2}:-m^{2}<\Lambda<-\frac{m^{2}}{3}$.

(c) The family (III) with $h>0$ (resp. $h<0$ ) in the region $R_{1}$ (resp. $\Omega_{1}$ ) is unstable (resp. linearly stable), while in the region $R_{2}$ (resp. $\Omega_{2}$ ) is linearly stable (resp. unstable).

(d) The two families (IV) with $h>0$ (resp. $h<0$ ) in the region $R_{3}$ (resp. $\Omega_{3}$ ) are linearly stable (resp. unstable), while in the region $R_{4}$ (resp. $\Omega_{4}$ ) are unstable (resp. linearly stable).

The proof of Theorem 3 is given in section 4 .

Kovalevskaya's idea and consequently Ziglin's and Morales-Ramis' theory go back to Poincaré (see Arnol'd [4]), who used the multipliers of the monodromy group of the variational equations associated to periodic orbits for studying the non-integrability of the differential equations. Poincaré's method allows to prove under convenient assumptions that the non Liouville-Arnol'd integrable systems have not any second first integral of class $\mathcal{C}^{1}$. See Appendix for more details. The main difficulty for applying the Poincaré non-integrability method to a given Hamiltonian system is to find for such a system periodic orbits having multipliers different from 1 . It seems that this result of Poincaré was forgotten by the mathematical community until modern Russian mathematicians (mainly Kozlov) have recently published on it, see $[4,13]$. Here we will apply the Poincaré criterion to the motion of our cosmological system (5), and we will show that either its motion is integrable and the two constants of motion have dependent gradients along the periodic orbits found in Theorem 1, or it is not Liouville-Arnol'd integrable with any second first integral of class $\mathcal{C}^{1}$. Of course for applying the Poincaré non-integrability theory to Hamiltonian systems (5), we need to study some of the periodic orbits of these systems and to compute their multipliers. For doing that we shall use the averaging theory, see the Appendix.

Our proof on the non Liouville-Arnol'd integrability uses isolated periodic orbits in the energy levels, the Holmes' proof (see [9]) uses transverse homoclinic orbits. It is important to remark that both our method and Holmes's method work for Hamiltonian systems which are close to integrable systems, but as we will see after a rescaling of 
the variables all systems (5) are close to integrable ones. The proof of Theorem 2 uses Theorem 1, and we obtain this last theorem perturbing an integrable Hamiltonian system, more precisely perturbing its quadratic part which of course is integrable.

Other interesting criterion on non-integrability also related with a Poincare's result was used by Meletlidou and Ichtiarouglou [16, 17, 18]. They consider perturbed Hamiltonian systems of the form $H=H_{0}+\varepsilon H_{1}$, where $H_{0}$ is a non-degenerate integrable Hamiltonian, and they show that some properties of the average value of the perturbing function $H_{1}$, evaluated along the non-isolated periodic orbits of $H_{0}$, are strongly connected with the non-integrability of the perturbed system. However this criterion cannot be applied to the Hamiltonian system (5) because it is degenerated.

There are two known cases where the Hamiltonian system (5) has an additional first integral, functionally independent of the Hamiltonian $H$. They were found by applying the so called ARS algorithm based on the Painlevé analysis [1]. The additional first integral of the system is

- $C=p_{1} p_{2}+\frac{1}{3}\left[m^{2}\left(q_{2}^{2}-q_{1}^{2}\right)-3\right]$ if $\lambda=\Lambda=-\frac{m^{2}}{3}$ with $m \neq 0$

- $C=q_{1} p_{2}+q_{2} p_{1}$ if $\lambda=\Lambda=-m^{2}$ with $m \neq 0$.

In the first integrable case $(\lambda, \Lambda)=\left(-\frac{m^{2}}{3},-\frac{m^{2}}{3}\right)=P_{1} \in l_{1} \cap l_{3}$, and in the second case the point $(\lambda, \Lambda)=\left(-m^{2},-m^{2}\right)=P_{3} \in l_{2} \cap l_{4}$. As we have mentioned after the statement of Theorem 1 in both points we cannot prove the existence of periodic solutions for the Hamiltonian system (5).

In [14] it is proved that the above two cases are the only meromorphically integrable cases of the Hamiltonian system (5) when $m \neq 0$. In fact, in order to prove the nonexistence of an additional meromorphic first integral on a non-zero Hamiltonian level the authors applied the Morales-Ramis theory (see [19] or [20]). Notice that the nonintegrability in this case is based in the non-existence of any additional meromorphic first integral in the sense of Liouville-Arnold. Our results are on the non-integrability in the sense of Liouville-Arnold for any second first integral of class $\mathcal{C}^{1}$. In [6] and [8] also the problem of non-existence of any additional meromorphic first integral in the Hamiltonian system (5) was considered.

Similar studies to the one done here for the cosmological coupled scalar field (5) about periodic orbits and their nonintegrability have been done for Yang-Mills, the Hénon-Heiles and the Armbruster-Guckenheimer-Kim Hamiltonians, see [10], [11] and [12], respectively.

\section{Proof of Theorem 1}

For proving Theorem 1 we shall apply Theorem 6 to the Hamiltonian system (5). Generically the periodic orbits of a Hamiltonian system with more than one degree of freedom are on cylinders fulfilled of periodic orbits. Therefore we cannot apply directly Theorem 6 to a Hamiltonian system, since the Jacobian of the function $f$ at the fixed point $a$ will be always zero. Then we must apply Theorem 6 to every Hamiltonian fixed level where the periodic orbits generically are isolated. Recall that the integrable Hamiltonian systems in the sense of Liouville-Arnol'd are non-generic, see [15].

On the other hand in order to apply Theorem 6 we need a small parameter $\varepsilon$. So in the Hamiltonian system (5) we change the variables $\left(q_{1}, q_{2}, p_{1}, p_{2}\right)$ to $\left(X, Y, p_{X}, p_{Y}\right)$ where $q_{1}=\sqrt{\varepsilon} X, q_{2}=\sqrt{\varepsilon} Y, p_{1}=\sqrt{\varepsilon} p_{X}$ and $p_{2}=\sqrt{\varepsilon} p_{Y}$. Since this change of 
coordinates is $\varepsilon^{-1}$-symplectic, the Hamiltonian function in these new variables assumes the form

$$
H=\frac{1}{2}\left(-p_{X}^{2}+p_{Y}^{2}\right)+\frac{1}{2}\left(-X^{2}+Y^{2}\right)+\varepsilon\left(\frac{m^{2}}{2} X^{2} Y^{2}+\frac{1}{4}\left[\Lambda X^{4}+\lambda Y^{4}\right]\right),
$$

and system (5) becomes

$$
\begin{aligned}
\dot{X} & =-p_{X}, \\
\dot{Y} & =p_{Y}, \\
\dot{p}_{X} & =X-\varepsilon\left(m^{2} X Y^{2}+\Lambda X^{3}\right), \\
\dot{p}_{Y} & =-Y-\varepsilon\left(m^{2} X^{2} Y+\lambda Y^{3}\right) .
\end{aligned}
$$

As the change of variables is only a scale transformation, for all $\varepsilon$ different from zero, the original and the transformed systems (5) and (8) have essentially the same phase portrait, and additionally system (8) for $\varepsilon$ sufficiently small is close to an integrable one

First we change the Hamiltonian (7) and the equations of motion (8) to convenient polar coordinates in such a way that for $\varepsilon=0$ we have a pair of harmonic oscillators. Thus we consider the change of variables

$$
X=r \cos \theta, \quad p_{X}=r \sin \theta, \quad Y=\rho \cos (-\theta+\alpha), \quad p_{Y}=\rho \sin (-\theta+\alpha),
$$

with $(r, \theta, \rho, \alpha) \in \mathbb{R}^{+} \times \mathbb{S}^{1} \times \mathbb{R}^{+} \times \mathbb{S}^{1}$. Recall that this is a change of variables when $r>0$ and $\rho>0$, also as this change of coordinates is not canonical, we lost the Hamiltonian structure of the differential equations. Moreover doing this change of variables appear in the system the angular variables $\theta$ and $\alpha$. Later on the variable $\theta$ will be used for obtaining the periodicity necessary for applying the averaging theory.

$$
h=\frac{1}{2}\left(-r^{2}+\rho^{2}\right)+\varepsilon\left(\frac{m^{2}}{2} r^{2} \rho^{2} \cos ^{2} \theta \cos ^{2}(-\theta+\alpha)+\frac{1}{4}\left[\Lambda r^{4} \cos ^{4} \theta+\lambda \rho^{4} \cos ^{4}(-\theta+\alpha)\right]\right),
$$

and the equations of motion are given by

$$
\begin{aligned}
\dot{r} & =-\varepsilon r \cos \theta \sin \theta\left[m^{2} \rho^{2} \cos ^{2}(-\theta+\alpha)+\Lambda r^{2} \cos ^{2} \theta\right] \\
\dot{\rho} & =-\varepsilon \rho \cos (-\theta+\alpha) \sin (-\theta+\alpha)\left[m^{2} r^{2} \cos ^{2} \theta+\lambda \rho^{2} \cos ^{2}(-\theta+\alpha)\right], \\
\dot{\theta} & =1-\varepsilon \cos ^{2} \theta\left[m^{2} \rho^{2} \cos ^{2}(-\theta+\alpha)+\Lambda r^{2} \cos ^{2} \theta\right], \\
\dot{\alpha} & =\varepsilon\left[-m^{2}\left(r^{2}+\rho^{2}\right) \cos ^{2} \theta \cos ^{2}(-\theta+\alpha)-\Lambda r^{2} \cos ^{4} \theta-\lambda \rho^{2} \cos ^{4}(-\theta+\alpha)\right] .
\end{aligned}
$$

Remember that the derivatives of the left hand side of these equations are with respect to the time variable $t$, which is not periodic. As for $\varepsilon$ sufficiently small $\dot{\theta}>0$ we can change to the $\theta$ variable as the independent one, and we denote by a prime the derivative with respect to $\theta$. The angular variable $\alpha$ cannot be used as the independent variable since the new differential system would not have the form (21) for applying Theorem 6 . 
Fixing the value of the first integral at $h$ such that $2 h+r^{2}>0$, and by solving equation (9) for $\rho$, and expanding $\rho$ in Taylor series of first order in $\varepsilon$ we obtain $(11)$

$$
\begin{aligned}
\rho= & \sqrt{2 h+r^{2}}+\varepsilon\left\{\frac{1}{32 \sqrt{2 h+r^{2}}}\right. \\
& {\left[-\left(2 h+r^{2}\right)\left(6 h \lambda+\left[4 m^{2}+3 \lambda\right] r^{2}\right)-3 \Lambda r^{4}-\left(\Lambda r^{4}+\lambda\left[2 h+r^{2}\right]^{2} \cos (4 \alpha)\right) \cos (4 \theta)\right.} \\
& -4\left(2 h+r^{2}\right)\left(2 h \lambda+\left[m^{2}+\Lambda\right] r^{2}\right) \sin (2 \theta) \sin (2 \alpha)-4 r^{2} \cos (2 \theta)\left(2 h m^{2}+\left(m^{2}+\Lambda\right) r^{2}\right. \\
& \left.+m^{2}\left(2 h+r^{2}\right) \sin (2 \alpha) \sin (2 \theta)\right)-2\left(2 h+r^{2}\right) \cos (2 \alpha)(2 \cos (2 \theta)[2 h \lambda \\
& \left.\left.\left.+\left(m^{2}+\lambda\right) r^{2}+m^{2} r^{2} \cos (2 \theta)\right]+m^{2} r^{2} \cos (2 \theta)+\lambda\left(2 h+r^{2}\right) \sin (2 \alpha) \sin (4 \alpha)\right)\right\} \\
& +O\left(\varepsilon^{2}\right) .
\end{aligned}
$$

If we write the differential equations of $r^{\prime}$ and $\alpha^{\prime}$ and substituting into the expression of $\rho$ given by (11), and expanding in Taylor series in powers of $\varepsilon$, we obtain the two differential equations

$$
\begin{aligned}
r^{\prime}= & -\varepsilon r \cos \theta \sin \theta\left[\Lambda r^{2} \cos ^{2} \theta+m^{2}\left(2 h+r^{2}\right) \cos ^{2}(-\theta+\alpha)\right]+O\left(\varepsilon^{2}\right), \\
\alpha^{\prime}= & \varepsilon\left[-\Lambda r^{2} \cos ^{4} \theta-\lambda\left(2 h+r^{2}\right) \cos ^{4}(-\theta+\alpha)\right. \\
& \left.-2 m^{2}\left(h+r^{2}\right) \cos ^{2} \theta \cos ^{2}(-\theta+\alpha)\right]+O\left(\varepsilon^{2}\right) .
\end{aligned}
$$

Clearly system (12) satisfies the assumptions of Theorem 6, and it has the form (21) with $F_{1}=\left(F_{11}, F_{12}\right)$, where

$$
\begin{aligned}
& F_{11}=-r \cos \theta \sin \theta\left[\Lambda r^{2} \cos ^{2} \theta+m^{2}\left(2 h+r^{2}\right) \cos ^{2}(-\theta+\alpha)\right], \\
& F_{12}=-\Lambda r^{2} \cos ^{4} \theta-\lambda\left(2 h+r^{2}\right) \cos ^{4}(-\theta+\alpha)-2 m^{2}\left(h+r^{2}\right) \cos ^{2} \theta \cos ^{2}(-\theta+\alpha) .
\end{aligned}
$$

The functions $F_{11}$ and $F_{12}$ are analytical. Furthermore they are $2 \pi$-periodic in the variable $\theta$, the independent variable of system (12). In order to apply the averaging theory of first order we must calculate the following averaged functions of $F_{11}$ and $F_{12}$

$$
\begin{aligned}
f_{1}(r, \alpha) & =\frac{1}{2 \pi} \int_{0}^{2 \pi} F_{11} d \theta \\
& =-\frac{m^{2}}{8} r\left(2 h+r^{2}\right) \sin (2 \alpha), \\
f_{2}(r, \alpha) & =\frac{1}{2 \pi} \int_{0}^{2 \pi} F_{12} d \theta \\
& =-\frac{1}{8}\left[h\left(4 m^{2}+6 \lambda\right)+r^{2}\left(4 m^{2}+3(\lambda+\Lambda)\right)+2 m^{2}\left(h+r^{2}\right) \cos (2 \alpha)\right] .
\end{aligned}
$$

We compute the real solutions $\left(r^{*}, \alpha^{*}\right)$ of $f_{1}(r, \alpha)=f_{2}(r, \alpha)=0$. It is important to remember that at order 0 in $\varepsilon$ we have $\rho=\sqrt{2 h+r^{2}}$. After some computations and since $r$ and $\rho$ cannot be simultaneously zero, we get the solutions:

(I) $r^{*}=0, \alpha^{*}= \pm \frac{1}{2} \arccos \left(-\frac{2 m^{2}+3 \lambda}{m^{2}}\right)$. Then $\rho^{*}=\sqrt{2 h}$ which give us the restriction $h>0$, and $m \neq 0$.

(II) $r^{*}=\sqrt{-2 h}, \alpha^{*}= \pm \frac{1}{2} \arccos \left(-\frac{2 m^{2}+3 \Lambda}{m^{2}}\right)$. Then $\rho^{*}=0$ and we have the restriction $h<0$ and $m \neq 0$.

(III) $r^{*}=\sqrt{-\frac{2 h\left(m^{2}+\lambda\right)}{2 m^{2}+\lambda+\Lambda}}, \alpha^{*}=0$. Then $\rho^{*}=\sqrt{\frac{2 h\left(m^{2}+\Lambda\right)}{2 m^{2}+\lambda+\Lambda}}$ we have the restriction $h\left(m^{2}+\lambda\right)\left(2 m^{2}+\lambda+\Lambda\right)<0$ and $h\left(m^{2}+\Lambda\right)\left(2 m^{2}+\lambda+\Lambda\right)>0$. 
(IV) $r^{*}=\sqrt{-\frac{2 h\left(m^{2}+3 \lambda\right)}{2 m^{2}+3(\lambda+\Lambda)}}, \alpha^{*}= \pm \frac{\pi}{2}$. Then $\rho^{*}=\sqrt{\frac{2 h\left(m^{2}+3 \Lambda\right)}{2 m^{2}+3(\lambda+\Lambda)}}$ we have the restriction $h\left(m^{2}+\Lambda\right)\left(2 m^{2}+3(\lambda+\Lambda)\right)<0$ and $h\left(m^{2}+3 \Lambda\right)\left(2 m^{2}+3(\lambda+\Lambda)\right)>0$.

Notice that in each solution $\left(r^{*}, \alpha^{*}\right)$ the Hamiltonian level $h$ must be non null.

Now we will check the Jacobian

$$
\left|D_{r, \alpha}\left(f_{1}, f_{2}\right)\right|_{\left(r^{*}, \alpha^{*}\right)}
$$

The Jacobian (14) of each solution (I) is

$$
\frac{3}{8} h^{2}\left(m^{2}+\lambda\right)\left(m^{2}+3 \lambda\right)
$$

which is not zero if $\lambda \neq-m^{2}$ and $\lambda \neq-\frac{m^{2}}{3}$. Therefore, if we eliminate the two straight lines $l_{1}$ and $l_{2}$ in the $(\lambda, \Lambda)$-plane, we guarantee the existence of these two families of periodic solutions (I) when $m \neq 0$ and $h>0$.

In case (II) the Jacobian is

$$
\frac{3}{4} h^{2}\left(m^{2}+\Lambda\right)\left(m^{2}+3 \Lambda\right)
$$

which is not zero if $\Lambda \neq-m^{2}$ and $\Lambda \neq-\frac{m^{2}}{3}$. Therefore if we eliminate the two straight lines $l_{3}$ and $l_{4}$ in the $(\lambda, \Lambda)$-plane, we guarantee the existence of these two families of periodic solutions (II) when $m \neq 0$ and $h<0$.

In case (III) the Jacobian is

$$
\frac{3}{4} m^{2} h^{2} \frac{\left(m^{2}+\lambda\right)\left(m^{2}+\Lambda\right)}{2 m^{2}+\lambda+\Lambda},
$$

which is not zero if $m \neq 0$ because the existence of the solution in this case already implies that $\frac{\left(m^{2}+\lambda\right)\left(m^{2}+\Lambda\right)}{2 m^{2}+(\lambda+\Lambda)} \neq 0$. In this case in the regions $R_{1}$ and $R_{2}$ we can guarantee the existence of this family of periodic solutions when $h>0$. Similarly if $h<0$ the existence of two periodic solutions is for the regions $\Omega_{1}$ and $\Omega_{2}$.

Finally for the case (IV) the Jacobian is equal to

$$
-\frac{3}{4} m^{2} h^{2} \frac{\left(m^{2}+3 \lambda\right)\left(m^{2}+3 \Lambda\right)}{2 m^{2}+3(\lambda+\Lambda)}
$$

as previously it is not zero if $m \neq 0$. Then in the regions $R_{3}$ and $R_{2}$ we can guarantee the existence of these two families of periodic solutions when $h>0$. Similarly if $h<0$ the existence of two periodic solutions is for the regions $\Omega_{3}$ and $\Omega_{4}$.

Summarizing, from Theorem 6 each solution of $f_{1}(r, \alpha)=f_{2}(r, \alpha)=0$ provides a periodic orbit of system (12) (and consequently of the Hamiltonian system (8) on the Hamiltonian level $h>0$ or $h<0$ according a carefully analysis of the previous solutions taking into account the lines $l_{1}, l_{2}, l_{3}, l_{4}$ and the regions $R_{1}, R_{2}, R_{3}, R_{4}, \Omega_{1}, \Omega_{2}, \Omega_{3}, \Omega_{4}$.

Notice that there are two periodic orbits if the solutions of case (I) exist; there are two periodic orbits if the solutions of case (II) exist; there is one periodic orbit if the solution of (III) exists, and there are two periodic orbits if the solutions of case (IV) exist. Now analyzing all the possible combinations, in fact, the possible intersections between the regions $R_{1}$ and $R_{3} ; R_{2}$ and $R_{4}$ and the half-lines $s_{i j}$ we obtain the conclusion for the case $h>0$. For example, if we take parameters in the region $R_{1} \cap R_{3}$ we have 5 periodic 
orbits and if we take parameters in the half-line $s_{13}$ we obtain the existence of only one periodic orbit (see Figure 1). In a similar way we analyze the case $h<0$ (see Figure 2).

In this way, we obtain the existence of one, two, three, fourth or five periodic orbits for positive and negative values of the Hamiltonian level $h$ if the solutions in case (I)-(IV) exists. Now the statements of Theorem 1 follow easily.

In the next section we will use the existence of these periodic orbits with multipliers different from 1 to study the non-integrability of the Hamiltonian system (8).

\section{Proof of Theorem 2}

We assume that we are under the assumptions of Theorem 1, and that one of the founded periodic orbits corresponding to the solutions of cases (I)-(IV) exist. Their associated Jacobians (15)-(18) are different from 1 playing with the energ level $h$. Since these Jacobians are the product of the four multipliers of these periodic orbits with two of them always equal to 1 , the remainder two multipliers cannot be equal to 1 . For more details on the computations of the multipliers see the last part of the Appendix. Hence under the assumptions of Theorem 1, by Theorem 5, either the conformal coupled field Hamiltonian systems cannot be Liouville-Arnol'd integrable with any second first integral $C$, or the system is Liouville-Arnol'd integrable and the differentials of $H$ and $C$ are linearly dependent on some points of these periodic orbits. Therefore the theorem is proved.

\section{Proof of Theorem 3}

For the family (I) we have that the eigenvalues of the averaged system (12) associated the point $\left(r^{*}=0, \alpha^{*}\right)$ are

$$
\lambda_{1}=-\sqrt{3} \pi \frac{h}{m^{2}} \sqrt{-\left(m^{2}+\lambda\right)\left(m^{2}+3 \lambda\right)}, \lambda_{2}=-\lambda_{1} .
$$

In particular, in the regions $I_{1}$ and $I_{3}$ the eigenvalues are imaginary pure so we can only affirm that the family of periodic orbits is linearly stable. While in the region $I_{2}$ the eigenvalues are real, then the family of periodic orbits is unstable.

In the case of the family (II) we have a similar result. In fact, the eigenvalues are

$$
\lambda_{1}=-\sqrt{3} \pi \frac{h}{m^{2}} \sqrt{-\left(m^{2}+\Lambda\right)\left(m^{2}+3 \Lambda\right)}, \lambda_{2}=-\lambda_{1} .
$$

In the regions $I I_{1}$ and $I I_{3}$ the family of periodic orbits is linearly stable. While in the region $I I_{2}$ the family of periodic orbits is unstable.

The eigenvalues of family (III) are

$$
\lambda_{1}=-\sqrt{3} \pi|h||m| \sqrt{-\frac{\left(m^{2}+\lambda\right)\left(m^{2}+\lambda\right)}{2 m^{2}+\lambda+\Lambda}}, \lambda_{2}=-\lambda_{1} .
$$

For $h>0$ (resp. $h<0$ ) in the region $R_{1}$ (resp. $\Omega_{1}$ ) the family of periodic orbits is unstable (resp. linearly stable) while in the region $R_{2}$ (resp. $\Omega_{2}$ ) is linearly stable (resp. unstable).

Finally, for the families (IV) the eigenvalues are

$$
\lambda_{1}=-\pi|h||m| \sqrt{\frac{\left(m^{2}+3 \lambda\right)\left(m^{2}+3 \lambda\right)}{2 m^{2}+3(\lambda+\Lambda)}}, \lambda_{2}=-\lambda_{1} .
$$


Thus for $h>0$ (resp. $h<0$ ) in the region $R_{3}$ (resp. $\Omega_{3}$ ) the family of periodic orbits is linearly stable (resp. unstable) while in the region $R_{4}$ (resp. $\Omega_{4}$ ) is unstable (resp. linearly stable).

In conclusion, we have proved Theorem 3 .

\section{Conclusions}

We have used two important tools in the area of dynamical systems. First the averaging method for studying the existence of periodic orbits and their stability of the Hamiltonian systems (5) in their Hamiltonian levels. The main results are summarized in Theorem 1 about the existence and in Theorem 3 about the type of stability of each family of periodic orbits. The second tool allows to study the non-integrability in the sense of Liouville-Arnol'd of the Hamiltonian systems (5), for any second first integral of class $\mathcal{C}^{1}$, see Theorem 2 .

It is important to remark that these two tools are based in the study of the periodic orbits via the averaging method, and that this method needs a small parameter, which is easy to obtain if we study Hamiltonian systems near integrable ones in the sense of Liouville-Arnol'd. However, the scale transformation introduced in the section 2 does not change the topology of the system, thus these results are valid for all $\varepsilon$, and in particular for $\varepsilon=1$. The two Hamiltonian systems (5) and (8) with $\varepsilon \neq 0$ have qualitatively the same phase portrait.

\section{Appendix}

6.1. Liouville-Arnol'd Theorem. In this section we summarize some facts on the Liouville-Arnol'd integrability of the Hamiltonian systems, and on the theory of the periodic orbits of the differential equations, for more details see $[3,5]$ and the subsection 7.1 .2 of [5], respectively. We present these results for Hamiltonian systems of two degrees of freedom, because we are studying a Hamiltonian system with two degrees of freedom associated to the motion of our Hamiltonian system associated for the conformally coupled scalar fields, but these results work for an arbitrary number of degrees of freedom.

We recall that a Hamiltonian system with Hamiltonian $H$ of two degrees of freedom is integrable in the sense of Liouville-Arnol'd if it has a first integral $C$ independent with $H$ (i.e. the gradient vectors of $H$ and $C$ are independent in all the points of the phase space except perhaps in a set of zero Lebesgue measure), and in involution with $H$ (i.e. the parenthesis of Poisson of $H$ and $C$ is zero). For Hamiltonian systems with two degrees of freedom the involution condition is redundant, because the fact that $C$ is a first integral of the Hamiltonian system, implies that the mentioned Poisson parenthesis is always zero. A flow defined on a subspace of the phase space is complete if its solutions are defined for all time.

Now we shall state the Liouville-Arnol'd Theorem restricted to Hamiltonian systems of two degrees of freedom.

Theorem 4. Suppose that a Hamiltonian system with two degrees of freedom defined on the phase space $M$ has its Hamiltonian $H$ and the function $C$ as two independent first integrals in involution. If $I_{h c}=\{p \in M: H(p)=h$ and $C(p)=c\} \neq \emptyset$ and $(h, c)$ is a regular value of the map $(H, C)$, then the following statements hold.

(a) $I_{h c}$ is a two dimensional submanifold of $M$ invariant under the flow of the Hamiltonian system. 
(b) If the flow on a connected component $I_{h c}^{*}$ of $I_{h c}$ is complete, then $I_{h c}^{*}$ is diffeomorphic either to the torus $\mathbb{S}^{1} \times \mathbb{S}^{1}$, or to the cylinder $\mathbb{S}^{1} \times \mathbb{R}$, or to the plane $\mathbb{R}^{2}$. If $I_{h c}^{*}$ is compact, then the flow on it is always complete and $I_{h c}^{*} \approx \mathbb{S}^{1} \times \mathbb{S}^{1}$.

(c) Under the hypothesis (b) the flow on $I_{h c}^{*}$ is conjugated to a linear flow on $\mathbb{S}^{1} \times \mathbb{S}^{1}$, on $\mathbb{S}^{1} \times \mathbb{R}$, or on $\mathbb{R}^{2}$.

The main result of this theorem is that the connected components of the invariant sets associated with the two independent first integrals in involution are generically submanifolds of the phase space, and if the flow on them is complete then they are diffeomorphic to a torus, a cylinder or a plane, where the flow is conjugated to a linear one.

Using the notation of Theorem 4 when a connected component $I_{h c}^{*}$ is diffeomorphic to a torus, either all orbits on this torus are periodic if the rotation number associated to this torus is rational, or they are quasi-periodic (i.e. every orbit is dense in the torus) if the rotation number associated to this torus is not rational.

We consider the autonomous differential system

$$
\dot{x}=f(x),
$$

where $f: U \rightarrow \mathbb{R}^{n}$ is $C^{2}, U$ is an open subset of $\mathbb{R}^{n}$ and the dot denotes the derivative respect to the time $t$. We write its general solution as $\phi\left(t, x_{0}\right)$ with $\phi\left(0, x_{0}\right)=x_{0} \in U$ and $t$ belonging to its maximal interval of definition.

We say that $\phi\left(t, x_{0}\right)$ is $T$-periodic with $T>0$ if and only if $\phi\left(T, x_{0}\right)=x_{0}$ and $\phi\left(t, x_{0}\right) \neq x_{0}$ for $t \in(0, T)$. The periodic orbit associated to the periodic solution $\phi\left(t, x_{0}\right)$ is $\gamma=\left\{\phi\left(t, x_{0}\right), t \in[0, T]\right\}$. The variational equation associated to the $T$ periodic solution $\phi\left(t, x_{0}\right)$ is

$$
\dot{M}=\left(\left.\frac{\partial f(x)}{\partial x}\right|_{x=\phi\left(t, x_{0}\right)}\right) M,
$$

where $M$ is an $n \times n$ matrix. The monodromy matrix associated to the $T$-periodic solution $\phi\left(t, x_{0}\right)$ is the solution $M\left(T, x_{0}\right)$ of $(20)$ satisfying that $M\left(0, x_{0}\right)$ is the identity matrix. The eigenvalues $\lambda$ of the monodromy matrix associated to the periodic solution $\phi\left(t, x_{0}\right)$ are called the multipliers of the periodic orbit.

For an autonomous differential system, one of the multipliers is always 1 , and its corresponding eigenvector is tangent to the periodic orbit.

A periodic solution of an autonomous Hamiltonian system always has two multipliers equal to one. One multiplier is 1 because the Hamiltonian system is autonomous, and another is 1 due to the existence of the first integral given by the Hamiltonian.

Theorem 5. If a Hamiltonian system with two degrees of freedom and Hamiltonian $H$ is Liouville-Arnol'd integrable, and $C$ is a second first integral such that the gradients of $H$ and $C$ are linearly independent at each point of a periodic orbit of the system, then all the multipliers of this periodic orbit are equal to 1.

Theorem 5 is due to Poincaré [21], section 36. It gives us a tool to study the non Liouville-Arnol'd integrability, independently of the class of differentiability of the second first integral. The main problem for applying this theorem is to find periodic orbits having multipliers different from 1. 
6.2. Averaging Theory of First Order. Now we shall present the basic results from averaging theory that we need for proving the results of this paper.

The next theorem provides a first order approximation for the periodic solutions of a periodic differential system, for the proof see Theorems 11.5 and 11.6 of Verhulst [23].

Consider the differential equation

$$
\dot{\mathbf{x}}=\varepsilon F_{1}(t, \mathbf{x})+\varepsilon^{2} F_{2}(t, \mathbf{x}, \varepsilon), \quad \mathbf{x}(0)=\mathbf{x}_{0}
$$

with $\mathbf{x} \in D$, where $D$ is an open subset of $\mathbb{R}^{n}, t \geq 0$. Moreover we assume that both $F_{1}(t, \mathbf{x})$ and $F_{2}(t, \mathbf{x}, \varepsilon)$ are $T$-periodic in $t$. We also consider in $D$ the averaged differential equation

$$
\dot{\mathbf{y}}=\varepsilon f_{1}(\mathbf{y}), \quad \mathbf{y}(0)=\mathbf{x}_{0},
$$

where

$$
f_{1}(\mathbf{y})=\frac{1}{T} \int_{0}^{T} F_{1}(t, \mathbf{y}) d t .
$$

Under certain conditions, equilibrium solutions of the averaged equation turn out to correspond with $T$-periodic solutions of equation (21).

Theorem 6. Consider the two initial value problems (21) and (22). Suppose:

(i) $F_{1}$, its Jacobian $\partial F_{1} / \partial x$, its Hessian $\partial^{2} F_{1} / \partial x^{2}, F_{2}$ and its Jacobian $\partial F_{2} / \partial x$ are defined, continuous and bounded by a constant independent of $\varepsilon$ in $[0, \infty) \times D$ and $\varepsilon \in\left(0, \varepsilon_{0}\right]$.

(ii) $F_{1}$ and $F_{2}$ are $T$-periodic in $t$ ( $T$ independent of $\varepsilon$ ).

Then the following statements hold.

(a) If $p$ is an equilibrium point of the averaged equation (22) and

$$
\left.\operatorname{det}\left(\frac{\partial f_{1}}{\partial \mathbf{y}}\right)\right|_{\mathbf{y}=p} \neq 0
$$

then there exists a $T$-periodic solution $\varphi(t, \varepsilon)$ of equation $(21)$ such that $\varphi(0, \varepsilon) \rightarrow$ $p$ as $\varepsilon \rightarrow 0$.

(b) The stability or instability of the limit cycle $\varphi(t, \varepsilon)$ is given by the stability or instability of the equilibrium point $p$ of the averaged system (22). In fact the singular point $p$ has the stability behavior of the Poincaré map associated to the limit cycle $\varphi(t, \varepsilon)$.

We point out the main facts in order to prove Theorem 6(b), for more details see section 6.3 and 11.8 in [23]. Suppose that $\varphi(t, \varepsilon)$ is a periodic solution of (21) corresponding to $y=p$ an equilibrium point of the averaged system (22). Linearizing equation (21) in a neighborhood of the periodic solution $\varphi(t, \varepsilon)$ we obtain a linear equation with $T$-periodic coefficients

$$
\dot{x}=\varepsilon A(t, \varepsilon) x, \quad A(t, \varepsilon)=\frac{\partial}{\partial x}\left[\left.F\left({ }_{1}(t, x)-F_{2}(t, x, \varepsilon)\right]\right|_{x=\varphi(t, \varepsilon)} .\right.
$$

We introduce the $T$-periodic matrices

$$
B(t)=\frac{\partial F_{1}}{\partial x}(t, p), \quad B_{1}=\frac{1}{T} \int_{0}^{T} B(t) d t, \quad C(t)=\int_{0}^{t}\left(B(s)-B_{1}\right) d s .
$$

From Theorem 6 we have

$$
\lim _{\varepsilon \rightarrow 0} A(t, \varepsilon)=B(t),
$$


and it is clear that $B_{1}$ is the matrix of the linearized averaged equation. The matrix $C$ has average zero. The near identity transformation

$$
x \longmapsto y=(I-\varepsilon C(t)) x,
$$

permits to write $(23)$ as

$$
\dot{y}=\varepsilon B_{1} y+\varepsilon[A(t, \varepsilon)-B(t)] y+O\left(\varepsilon^{2}\right) .
$$

Notice that $A(t, \varepsilon)-B(t) \rightarrow 0$ as $\varepsilon \rightarrow 0$, and also the characteristic exponents of equation (25) depend continuously on the small parameter $\varepsilon$. It follows that, for $\varepsilon$ sufficiently small, if the determinant of $B_{1}$ is not zero, then 0 is not an eigenvalue of the matrix $B_{1}$ and then it is not a characteristic exponent of (25). By the near-identity transformation we obtain that system (23) has not multipliers equal to 1.

\section{ACKNOWLEDGMENTS}

The first author is partially supported by the grants MEC/FEDER MTM 200803437, CIRIT 2009SGR 410 and ICREA Academia. The second author is partially supported by Fondecyt 1080112.

\section{REFERENCES}

[1] Ablowitz M., Ramani A. and Segur H., A connection between nonlinear evolution equations and ordinary differential equations of P-type I, J. Math. Phys. 27 (1980), 15-721.

[2] Almeida M.A., López-Castillo A. and Stuchi T.J., Non-integrability proof of the frozen planetary atom configuration, J. Physics A: Math. and Gen. 36 (2003), 4805-4814.

[3] Abraham R. and Marsden J.E., Foundations of Mechanics, Benjamin, Reading, Masachusets, 1978.

[4] Arnol'd V.I., Forgotten and neglected theories of Poincaré, Russian Math. Surveys 61 No. 1 (2006), $1 \hat{\mathrm{A}}-18$.

[5] Arnol'd V.I., Kozlov V. and Neishtadt A., Dynamical Systems III. Mathematical Aspects of Classical and Celestial Mechanics, Third Edition, Encyclopaedia of Mathematical Science, Springer, Berlin, 2006.

[6] Boucher D.and Weil J., About non-integrability in the Friedmann-Robertson-Walker cosmological model, Brazilian J. Phys., 37 A (2007), 398-405.

[7] Buică A. and Llibre J., Averaging methods for finding periodic orbits via Brouwer degree, Bull. Sci. Math. 128 (2004), 7-22.

[8] Coelho L., Skea A. and Stuchi T., On the integrability of Friedmann-Robertson-Walker models with conformally coupled massive scalar fields, J. Phys. A: Math. Theor. 41 (2008) 075401.

[9] Holmes P., Proof of non-integrability for the Hénon-Heiles Hamiltonian near an exceptional integrable case, Physica D: Nonlinear Phenomena 5 (1982), 335-347.

[10] Jiménez-Lara L. and Llibre J., Periodic orbits and nonintegrability of generalized classical YangMills Hamiltonian systems, J. Math. Physics 52 (2011), 032901.

[11] Jiménez-Lara L. and Llibre J., Periodic orbits and Hénon-Heiles systems, J. Phys. A: Math. Theor. 44 (2011), 205103-14pp.

[12] Llibre J. and Roberto L., Periodic orbits and non-integrability of Armbruster-Guckenheimer-Kim potential, submitted.

[13] Kozlov V.V., Integrability and non-integrability in Hamiltonian mechanics, Russian Math. Surveys 38 No. 1 (1983), 1-76.

[14] Maciejewski A., Przybylska M., Stachowiak T., and Szydłowski M., Global integrability of cosmological scalar fields, J. Phys. A: Math. Theor. 41 (2008), 465101.

[15] Markus L. and Meyer, K.R., Generic Hamiltonian Dynamical Systems are neither integrable nor ergodic, Memoirs of the Amer. Math. Soc. 144, 1974.

[16] Meletlidou E. and Ichtiaroglou S., A criterion for non-integrability based on Poincaré's theorem, Physica D 71 (1994), 261-268.

[17] Meletlidou E. and Ichtiaroglou S., On the number of isolating integrals in perturbed Hamiltonian system with $n \geq 3$ degrees of freedom, J. Phys. A: Math. Gen. 27 (1994), 3919-3926. 
[18] Meletlidou E., Stagika G. and Ichtiaroglou S., Non-integrability and structure of the resonance zones in a class of galactic potentials, Cel. Mech. and Dyn. Astron. 91 (2005), 323-335.

[19] Morales-Ruiz J.J., Differential Galois Theory and non-integrability of Hamiltonian systems, Progress in Math. Vol. 178, Birkhauser, Verlag, Basel, 1999.

[20] Morales-Ruiz J.J., Ramis J.P. and Simó C., Integrability of Hamiltonian Systems and Differential Galois Groups of Higher Variational Equations, Annales Scientifiques de 1Õ̂Acole Normale SupÂrieure, 40, (2007), 845-884

[21] Poincaré H., Les méthodes nouvelles de la mécanique céleste, Vol. I, Gauthier-Villars, Paris 1899.

[22] J.A. Sanders, F. Verhulst and J. Murdock, Averaging Methods in Nonlinear Dynamical Systems, Appl. Math. Sci. 59, 2nd. ed., Springer, Berlin, 2007.

[23] Verhulst F., Nonlinear Differential Equations and Dynamical Systems, Universitext, Springer, 1991.

${ }^{1}$ Departament de Matemàtiques, Universitat Autònoma de Barcelona, 08193 Bellaterra, Barcelona, Catalonia, Spain

E-mail address: jllibre@mat.uab.cat

2 Departamento de Matemática, Universidad del Bio Bio, Concepción, Avda. Collao 1202, CHILE

E-mail address: clvidal@ubiobio.cl 